\title{
Prevention of neointimal formation after angioplasty using tetramethylpyrazine-coated balloon catheters in a rabbit iliac artery model
}

\author{
Lijuan Chen ${ }^{1}$, Si Pang ${ }^{1}$, Chunshu Hao ${ }^{1}$, Aiming Xie ${ }^{1}$, Kongbo Zhu ${ }^{1}$, Yanru He ${ }^{1}$, Xiaoguo Zhang ${ }^{1}$, \\ Wenbing Lu' ${ }^{1}$, Genshan $\mathrm{Ma}^{1}$, Zhong Chen ${ }^{2,3}$ \\ ${ }^{1}$ Department of Cardiology, Zhongda Hospital Affiliated to Southeast University, Nanjing 210009, China; ${ }^{2}$ Department of Cardiology, Shanghai \\ University of Medicine and Health Sciences Affiliated Sixth People's Hospital East, Shanghai 201306, China; ${ }^{3}$ Department of Cardiology, Shanghai \\ Jiao Tong University Affiliated Sixth People's Hospital, Shanghai 200233, China \\ Contributions: (I) Conception and design: Z Chen; (II) Administrative support: None; (III) Provision of study materials or patients: Z Chen, G Ma; \\ (IV) Collection and assembly of data: C Hao, A Xie, K Zhu, Y He, X Zhang, W Lu; (V) Data analysis and interpretation: L Chen; (VI) Manuscript \\ writing: All authors; (VII) Final approval of manuscript: All authors. \\ Correspondence to: Zhong Chen. Department of Cardiology, Shanghai University of Medicine and Health Sciences Affiliated Sixth People's Hospital \\ East, Shanghai Jiao Tong University Affiliated Sixth People’s Hospital, China. Email: zhongchen7498@hotmail.com; Genshan Ma. Department of \\ Cardiology, Zhongda Hospital Affiliated to Southeast University, China. Email: magenshan@seu.edu.cn.
}

Background: Restenosis remains a clinical problem; drug-coated balloons (DCBs) have demonstrated high efficiency in this situation. DCBs prevent neointimal hyperplasia by inhibiting cell proliferation and migration. Tetramethylpyrazine (TMP) is a traditional Chinese medicine originally isolated from the rhizome of Ligusticum Walliichii, which can inhibit platelet aggregation and smooth muscle cell proliferation. We hypothesized that TMP-coated balloons (TCB) could reduce neointimal hyperplasia through the NF- $\kappa \mathrm{B}$ signalling pathway.

Methods: Twenty-one New-Zealand White rabbits $(2.5-3.0 \mathrm{~kg}$, male) were fed high-fat diets; 36 bilateral iliac artery stenosis models were successfully established by balloon straining. Rabbits were randomly treated with TCB ( $\mathrm{n}=20)$ or plain balloons (PBA, $\mathrm{n}=16$ ) (3 died during model construction). Angiographies were recorded at baseline, the immediate period, and 4 weeks later. Animals were euthanized and arteries collected for histological analysis and immunohistochemical staining. Protein expression of proliferating cell nuclear antigen (PCNA) and nuclear factor kappa-light-chain-enhancer of activated B cells (NF- $\kappa \mathrm{B}) \mathrm{p} 65$ of the vessel samples were analyzed using Western blotting.

Results: No difference existed in the baseline lesion characteristics or procedural results. Angiographic follow-up was successfully performed on 18 rabbits (TCB: $n=20$, PBA: $n=16$ ), except for 3 deaths related to the operation. Treatment with TCB was superior to that with PBA, with lower late lumen loss $(0.45 \pm 0.23 v s$. $0.84 \pm 0.17 \mathrm{~mm}, \mathrm{P}<0.01)$. Pathological analysis confirmed the efficiency of TCB through decreasing the area stenosis rate compared with $\mathrm{PBA}(46.48 \% \pm 8.22 \%$ vs. $75.24 \% \pm 6.10 \%, \mathrm{P}<0.01)$. As determined by Western blotting, significant reductions occurred in PCNA and NF- $\kappa$ B p65 protein intensity in the TCB group versus the PBA group (all $\mathrm{P}<0.01$ ). TCB efficiently mitigated restenosis in the rabbit iliac artery model.

Conclusions: This study elucidated that TCB could restrain intimal hyperplasia of vessels by inhibiting the activation of the NF- $\mathrm{BB}$ pathway to reduce inflammatory response and decrease the rate of cell proliferation through suppressing PCNA expression.

Keywords: Tetramethylpyrazine (TMP); restenosis; inflammatory; proliferation; prevention

Submitted Jun 22, 2019. Accepted for publication Aug 22, 2019.

doi: $10.21037 / \mathrm{cdt} .2019 .09 .04$

View this article at: http://dx.doi.org/10.21037/cdt.2019.09.04 


\section{Introduction}

Percutaneous transluminal coronary angioplasty (PTCA) has become the most accepted therapy for coronary artery disease (CAD). However, restenosis remains a significant problem in the PTCA era. The restenosis rate has been as high as $30-50 \%$ in patients who received plain balloon angioplasty (1), and bare metal stent implantation reduced the rate by $10 \%$ (2), which imposed great suffering on patients (3). Research shows that the mechanisms of restenosis include initial elastic recoil, negative remodeling, neointimal hyperplasia, and inflammatory response $(2,4)$. The common factor driving the inflammatory response is the transcription factor NF- $\mathrm{KB}$. Research shows that inflammation mediated by the NF- $\mathrm{\kappa B}$ signalling pathway is involved in postangioplasty.

Clinical evidence has shown that drug-eluting stents (DES) have significantly reduced restenosis via inhibiting intimal hyperplasia. Drug-coated balloons (DCB) could be a prioritized alternative to cover the limitations of DES. First, DCB can homogeneously release drugs to local vessel walls quickly and maintain highest drug concentrations at injury time, with a greater effect on initial neointimal response. Second, chronic inflammation and thrombosis are decreased due to the absence of stents, leading to a shorter duration of dual antiplatelet therapy (5).

One type of drug carried by DCBs is the antiproliferative drug, mostly paclitaxel. SeQuent Please and DIOR are used widely in the world with certain efficacy, but the cost is too high for most patients to afford. Moreover, the Chinese owned DCB invented independently has not been marketed (6-8). Therefore, it is necessary to develop an efficient, cheap, and safe DCB. Tetramethylpyrazine (TMP) is a traditional Chinese medicine originally isolated from the rhizome of Ligusticum Walliichii, which can inhibit platelet aggregation and smooth muscle cell proliferation. A sterile injectable solution of TMP has been widely applied for treatment of $\mathrm{CAD}$, cerebral infarction, peripheral vascular occlusion, and complications of diabetes mellitus. Our previous study has suggested that TMP-eluting stents (TES) can restrain intimal hyperplasia in the porcine coronary artery (9). Therefore, we hypothesized that a TMP-coated balloon (TCB) could reduce neointimal hyperplasia through the NF- $\kappa \mathrm{B}$ signalling pathway.

\section{Methods}

\section{Ethics statement}

The animal experiments were approved by the institutional animal care and use committee of Southeast University. All animal experiments in this study comply with the ARRIVE guidelines and are carried out in accordance with the U.K. Animals (Scientific Procedures) Act, 1986, and associated guidelines, EU Directive 2010/63/EU for animal experiments.

\section{Animal grouping and feeding}

Twenty-one numbered male New Zealand White rabbits $(2.5-3.0 \mathrm{~kg})$ with 36 numbered iliac arteries were randomly assigned to different study subgroups [stenotic iliac arteries $(\mathrm{n}=36) ; 3$ animals died during the operation]. The animals were housed individually and fed with a $1 \%$ cholesterol diet and free access to water for 1 week prior to the operation. Oral aspirin $(100 \mathrm{mg} /$ day $)$ for antiplatelet effect was fed from 1 day before the procedure to the end of the study. All animals were cared for professionally at the animal experiment center of Southeast University.

\section{Preoperative preparation and postoperative care}

A venous indwelling needle was established under the vein of the rabbit ear. The animal received $1 \%$ pentobarbital sodium ( $2 \mathrm{~mL} / \mathrm{kg}$, intravenous injection) for general anaesthesia, and $2 \%$ lidocaine (subcutaneous injection) was supplemented for topical anaesthesia. The rabbits were kept in a supine position to open the airway, with assisted respiration performed with mask oxygen inhalation. Carefully observing the breathing performance is the key point of intraoperative safety. Moreover, to prevent periprocedural bacteraemia, penicillin (80 million units/day) was injected intramuscularly during the postoperative period (on the operative day 0 , and postoperative day 1 and day 2 ).

\section{Preparation of TMP-coated balloons}

The sterile solution was made up of a mixture of $3 \mathrm{~g}$ TMP and $10 \mathrm{~mL}$ of ethyl alcohol. A $(2.5-2.75) \mathrm{mm} \times 20 \mathrm{~mm}$ balloon catheter was put into an ultrasonic sprayer dedicated for drug balloons, and then the spraying procedure began after the balloon was tightly strained and inflated. During the spraying process with the ultrasonic power of the sprayer of $120 \mathrm{kHz}$ and homogeneous atomized particles of the solution of 10-20 $\mu \mathrm{m}$, the drug balloon moved longitudinally and rotated circumferentially. After that, the sprayed balloon was put into a balloon folding machine to assemble the mould. In the end, the balloon was covered 
Table 1 Quantitative analysis iliac angiography post treatment in both groups at day 56

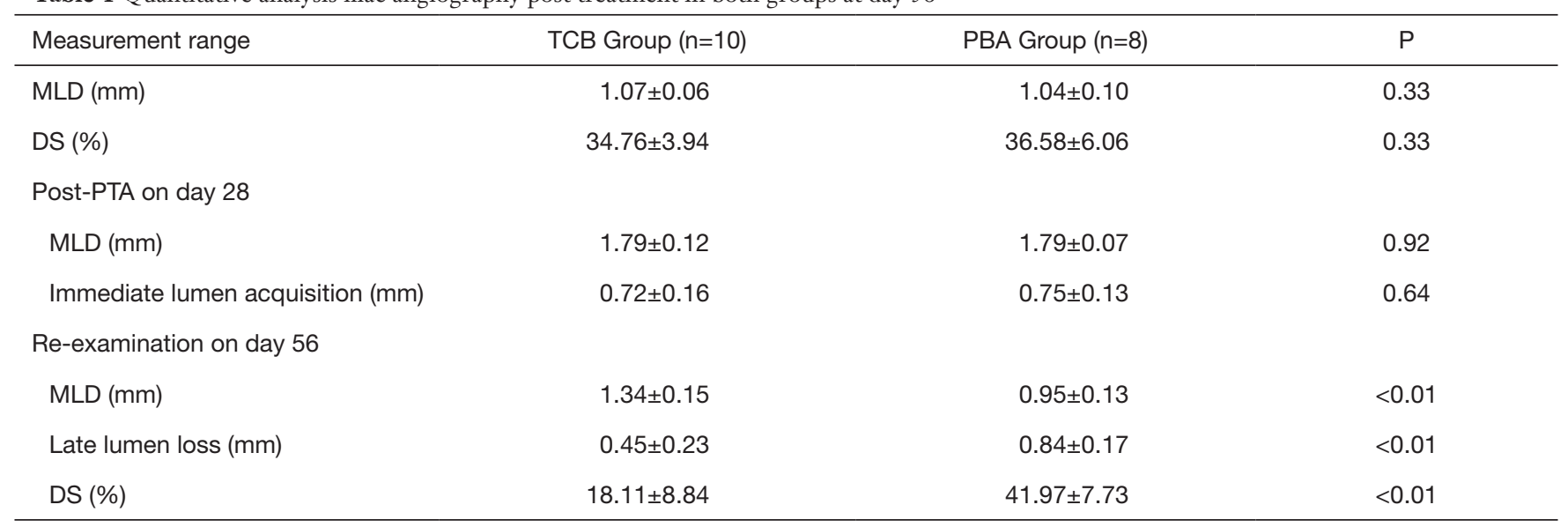

Values are presented as mean \pm SD. $n$ represents the number of rabbits. P $>0.05$ shows no significance. RVD, reference vessel diameter; MLD, minimal lumen diameter; DS, \% diameter stenosis; PTA, percutaneous transluminal angioplasty.

with a protective sleeve and placed on a tube plate. Drug content was measured by using a gravimetric test. No weight gain occurred when the balloon was sprayed 50 laps; however, $30 \mu \mathrm{g}$ of drug was loaded when 100 laps were sprayed.

\section{Development of iliac atherosclerotic stenosis model by balloon-injury at day 0}

Preoperative fasting 8 hours before operation played an important role in increasing the survival rate. General anaesthesia was constructed as described above. Then the rabbit was fixed on the dedicated operating the supine position and the leg extension status was maintained. The inguinal area was disinfected by wiping with iodophor disinfectant solution 3 times; strictly obeying asepsis operation rules reduced the risk of infection. The femoral artery pulsation was touched to determine its anatomical position, and then $2 \%$ lidocaine was injected locally. First, the subcutaneous skin tissue, muscle, and fascia were cut approximately $4 \mathrm{~cm}$ in length, to expose the femoral artery, femoral vein, and femoral nerve. Constant vigilance was necessary to avoid damage to the nervous system. After sufficient dissection and isolation of the femoral artery for $2 \mathrm{~cm}$ in length, two 3-0 suture silk threads were used for ligation of the distal end of the artery, and the proximal end was clamped with a vascular clip. Eliminating operative haemorrhage was important to minimize the mortality and disability rate of the operation. Careful puncture was performed through a small opening cut in the femoral artery to insert the microcatheter and 0.014-inch guide wire; then, heparin $(120 \mathrm{U} / \mathrm{kg})$ was promptly injected through the vein. Delivery of the angioplasty balloon catheter into the iliac artery was followed under fluoroscopic guidance. The 10 -atm pressure balloon angioplasty was performed, and repeated pulling back and pushing forward was conducted 3 times to develop vascular endothelial damage. After the femoral artery was ligatured, the incision was sutured layer by layer. The rabbits were fed a high-fat diet after the first period operation for 4 weeks.

\section{Percutaneous transluminal iliac angioplasty through the common carotid artery at day 28}

To further investigate the efficacy of TCB catheter inhibition of intimal hyperplasia in a clinically relevant situation, the stenotic lesions were confirmed through angiography four weeks after initial endothelial denudation. All surviving animals $(\mathrm{n}=18, \mathrm{~N}=36)$ were randomly divided into two subgroups (TCB group: $\mathrm{n}=10, \mathrm{~N}=20$; PBA group: $\mathrm{n}=8 ; \mathrm{N}=16$ ) (10). Similar preoperative preparation, anaesthesia, and disinfection were carried out. The common carotid arteries were isolated, and the guide wire was inserted. Then, heparin $(120 \mathrm{U} / \mathrm{kg})$ was injected to prevent thrombus formation. Under fluoroscopic guidance, the 0.014-inch coronary guide wire, microcatheter, and 5-in-6 catheter were advanced from the carotid artery into the descending aorta at the first or second intercostal level. Angiogram sequences were recorded after the injection of contrast radiography. The vascular stenosis was observed 
and then a balloon catheter was inserted into the narrowest lesion and inflated with a pressure of 10 atm for 1 minute, according to the reference vessel diameter (RVD). Then, angiography was conducted again. Immediately after the procedure, no complications occurred, or no residual stenosis was observed on the iliac artery angiography. The silks were tied tightly upon the removal of the introducer sheath, and muscle, fascia, and skin were then sutured with 2-0 black silk. After normal use of antibiotics intramuscularly, the rabbits were returned to the animal facility safely and fed with a high-fat diet for another 4 weeks.

\section{Angiographic analysis}

Surviving animals were observed for another 4 weeks and then received another angiography before planned death. The surgical procedures for angiographic examination were mostly similar to the above steps, except that the left carotid artery was used as the access route. QAngio-XA Software (Medis, Medical Imaging Systems, Leiden, the Netherlands) was used to analyse the angiograms of immediate post-PTBA and 28 days after PTBA to measure the minimal lumen diameter (MLD), RVD, and diameter of stenosis [DS\% = $(1-\mathrm{MLD}) / \mathrm{RVD} \times 100 \%$ ]. Late lumen loss was then calculated (MLD-immediate post-PTA - MLD-28 days after PTA).

\section{Histological analysis}

The 1.5-2-cm segments of both arteries were dehydrated by using ethyl alcohol, embedded in paraffin to preserve vessel integrity, cut into 5 cross-sectional segments $4-\mu \mathrm{m}$ thick, and stained with haematoxylin and eosin for histomorphological analysis. Haematoxylin and eosin staining clearly showed the internal elastic lamina, the external elastic lamina, and the neointima. Each histologic section was scanned, and the intimal and external elastic lamina were manually identified. Luminal area (LA), luminal perimeter (LP), internal elastic membrane area (IEMA), and external elastic membrane area (EEMA) were measured using Image-Pro Plus version 6.0 (Media Cybernetics, USA) to obtain the neointimal area (NA), tunica medial area (MA), and area of stenosis (AS\%).

\section{Western blotting}

Iliac arteries were first lysed in lysis buffer $(720 \mu \mathrm{L}$ of RIPA,
$20 \mu \mathrm{L}$ of PMSF, $100 \mu \mathrm{L}$ of complete protease inhibitor, $100 \mu \mathrm{L}$ of Phos-stop, $50 \mu \mathrm{L}$ of $\mathrm{NaF}$, and $10 \mu \mathrm{L}$ of Na3VO4). Soluble extracts were incubated on ice for $15 \mathrm{~min}$, followed by centrifugation at $14,000 \times \mathrm{g}$ for $30 \mathrm{~min}$ at $4{ }^{\circ} \mathrm{C}$. After centrifugation of the samples, the protein concentrations were determined using the BCA Protein Assay Kit (Pierce, Thermo Fisher Scientific, United States). The lysates $(50 \mu \mathrm{g})$ were separated by SDS/PAGE (Invitrogen, Thermo Fisher Scientific, United States) and transferred to a PVDF membrane (Millipore, United States). After blocking with $5 \%(\mathrm{w} / \mathrm{v})$ non-fat dried skimmed milk powder for $1 \mathrm{~h}$ at room temperature, membranes were incubated with the specific primary antibodies overnight at $4^{\circ} \mathrm{C}$. After incubation with the horseradish peroxidase-conjugated secondary antibodies for $1 \mathrm{~h}$ at room temperature, immunoblots were revealed by using ChemiDocTM XRS + (Bio-Rad Laboratories, United States). Expression levels of specific proteins were normalized to actin on the same membrane.

The band detection was performed with a colorimetric Western blotting visualization reagent. The images were processed with Adobe Photoshop and analysed with an Alpha Software Processing System to obtain grey values of the bands.

\section{Statistical analysis}

SPSS 20.0 software was used to process data, presented as the means \pm standard deviation. Normality test was performed to analyse the main indicators, whereas the $t$-test was used to detect differences between TCB and PBA subgroups. A value of $\mathrm{P}<0.05$ was considered statistically significant.

\section{Results}

\section{Development of iliac artery restenosis model}

After the iliac artery injury model was established by balloon dilatation, to confirm the successful establishment of the iliac artery stenosis model, two rabbits were sacrificed 7, 14, and 28 days after iliac artery injury, respectively. Bilateral iliac arteries were sectioned with haematoxylin eosin $(\mathrm{HE})$ staining. Figure 1 shows mild hyperplasia of the intima and clear demarcation of the vascular layers in the iliac artery of the rabbit at 7 days. Moderate hyperplasia of the local endometrium and disorderly arrangement of fibrocytes in the mesenchyma with cell deficiency were observed at 

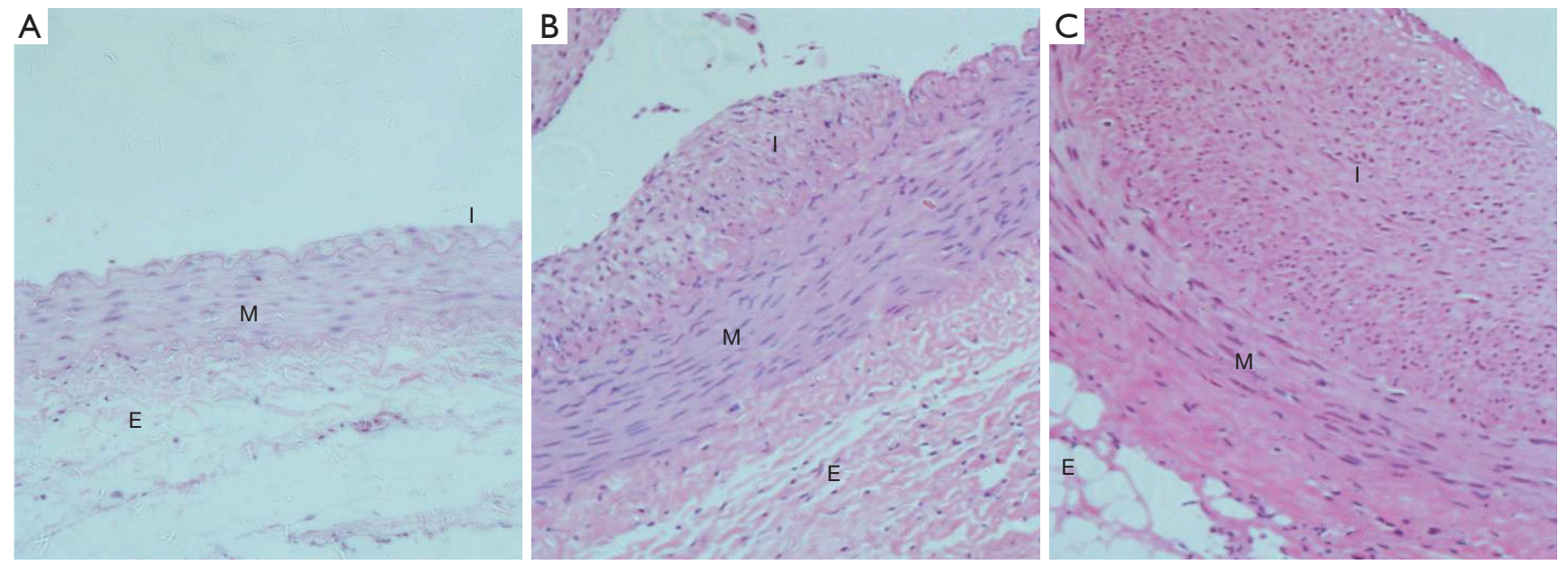

Figure 1 Hematoxylin eosin (HE) staining showed the iliac artery injury after balloon dilatation on day 7, 14, 28 ( $\times 200)$. (A) Iliac artery injury on 7 days; (B) iliac artery injury on 14 days; (C) iliac artery injury on 28 days. I, intimal membrane; M, medial membrane; E, external membrane.

14 days. Severe endometrial hyperplasia was observed in the lumen with visible fibrous cap at 28 days.

\section{Inbibitory effects of TCB catheter on neointimal formation after angioplasty through QCA and histopathological analysis}

Pre-angioplastic angiograms showed a similar DS\% between the TCB group and PBA group $(\mathrm{P}>0.05)$ and immediate-postangiographic angiograms did not visually show obvious residual stenosis of the lumen. On day 56, iliac artery stenosis was reduced in the TCB group but not in the PBA group.

Quantitative angiographic analysis obtained the results exhibited in Table 1. On day 28, no differences were noted in MLD or DS\% between the two groups anteroposterior angioplasty. On day 56, in comparison with the PBA group, MLD was larger in the TCB group $(1.34 \pm 0.15 \mathrm{vs}$. $0.95 \pm 0.13 \mathrm{~mm}, \mathrm{P}<0.01)$, with smaller DS $\%(18.11 \% \pm 8.84 \%$ vs. $41.97 \% \pm 7.73 \%, \mathrm{P}<0.01)$.

On day 56 , all remaining rabbits were sacrificed to obtain lesion sections of iliac arteries. As shown in Figure 2, both $\mathrm{TCB}$ and $\mathrm{PBA}$ groups revealed neointimal hyperplasia microscopically. For the PBA group (Figure $2 A$ ), the arterial lumen was apparently narrow with significantly thicker neointimal formation. Elastic fibres were loose in the tunica media with a clear interface. The boundary between the tunica intima and media was unclear. At the same time, lumens in the TCB group (Figure 2B) had mild stricture. Neointima proliferated moderately and homogeneously, with a thinner average thickness of hyperplasia. The internal elastic membrane was wavy, showing a clear boundary between the tunica intima and media. Approximately 8 to 10 layers of smooth muscle cells composed the tunica media. Tunica externa consisted of loose connective tissues in both groups.

Quantitative analysis results of the pathological sections are listed in Table 2. In contrast to the PBA group, AS\% was significantly lower in the TCB group $(46.48 \% \pm 8.22 \%$ vs. $75.24 \% \pm 6.10 \%, \mathrm{P}<0.01)$, whereas $\mathrm{LA}$ and $\mathrm{LP}$ were markedly larger. Moreover, IEMA and EEMA in the TCB group tended to increase, which might be related to positive vascular remodelling.

\section{Molecular mechanisms underlying the prevention of neointimal formation by the NF- $\mathrm{KB}$ signalling pathway}

In the current study, the expression of a-SMA and proliferating cell nuclear-antigen was significantly decreased in the TCB group compared with the PBA group. To further investigate the molecular mechanisms by which TCB inhibited neointimal hyperplasia, we detected NF$\kappa \mathrm{B}$ expression at the protein level using Western blotting. Western blotting results are shown in Figure 3. Proliferating cell nuclear antigen (PCNA) and NF- $\mathrm{KB}$ were significantly decreased in the TCB group compared with the PBA 

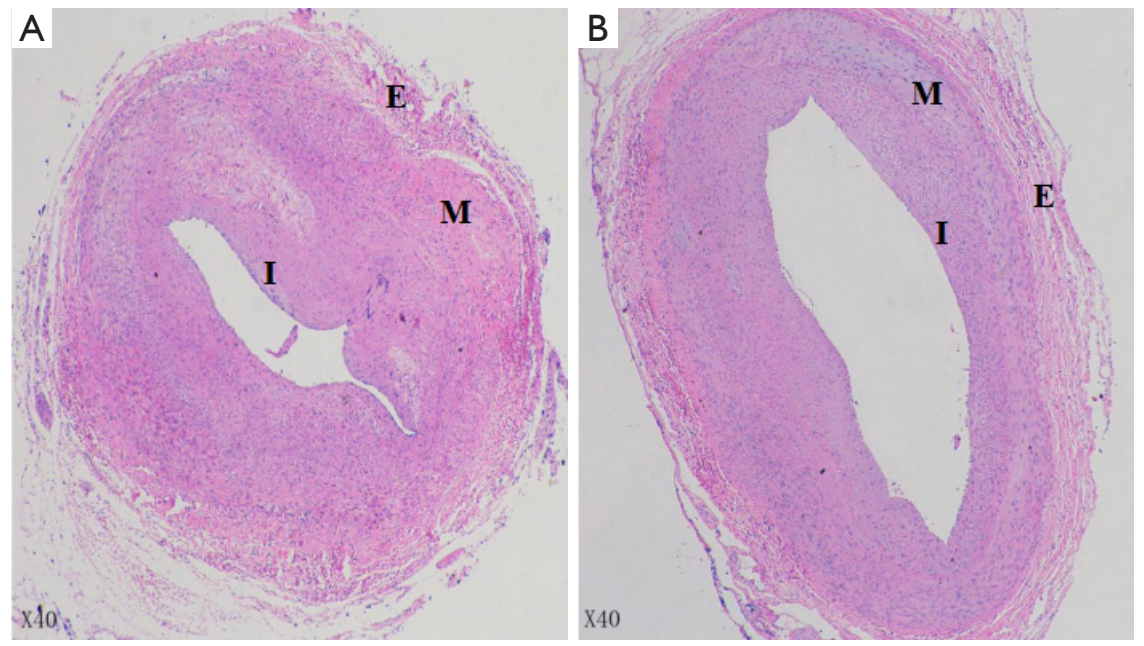

Figure 2 Results of HE staining histologic cross-sections of rabbit iliac artery at day 56 (×40). (A) PBA group; (B) TCB group, with clear internal elastic membrane and external elastic membrane. I, intimal membrane; $\mathrm{M}$, medial membrane; E, external membrane.

Table 2 Quantitative analysis of immunohistochemistry after treatment in both groups

\begin{tabular}{lccc}
\hline Measurement range & PBA group $(\mathrm{n}=10)$ & TCB group $(\mathrm{n}=8)$ & $\mathrm{P}$ \\
\hline IEMA $\left(\mathrm{mm}^{2}\right)$ & $0.62 \pm 0.05$ & $0.80 \pm 0.38$ & 0.08 \\
EEMA $\left(\mathrm{mm}^{2}\right)$ & $1.41 \pm 0.11$ & $1.59 \pm 0.33$ & 0.05 \\
NA $\left(\mathrm{mm}^{2}\right)$ & $0.47 \pm 0.02$ & $0.37 \pm 0.19$ & 0.08 \\
LA $\left(\mathrm{mm}^{2}\right)$ & $0.16 \pm 0.05$ & $0.42 \pm 0.21$ & $<0.01$ \\
LP $(\mathrm{mm})$ & $2.16 \pm 0.32$ & $3.31 \pm 0.71$ & $<0.01$ \\
AS $(\%)$ & $75.24 \pm 6.10$ & $46.48 \pm 8.22$ & $<0.01$ \\
\hline
\end{tabular}

Values are presented as mean \pm SD. $n$ represents the number of rabbits. $P>0.05$ shows no significance. IEMA, internal elastic membrane area; EEMA, external elastic membrane area; NA, neointimal area; LA, lumen area; LP, lumen perimeter; AS, \%area stenosis.
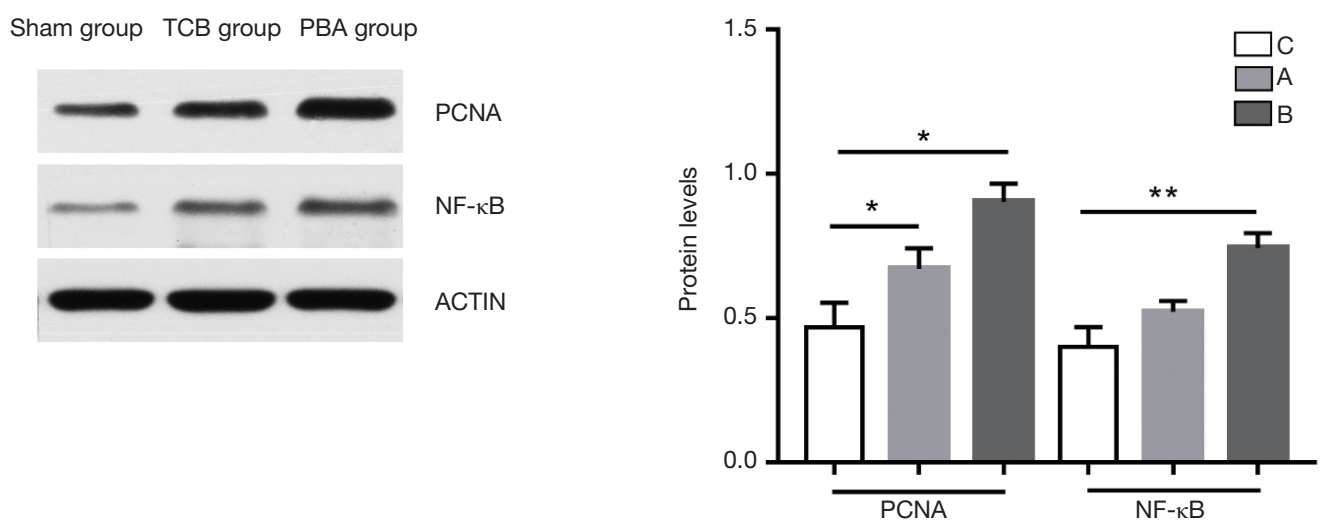

Figure 3 Western blotting shows PCNA and NF- $\kappa B$ were significantly decreased in the TCB group compared to the PBA group. * $\mathrm{P}<0.05$; **, $\mathrm{P}<0.01$. 
group. This indicates that TCB could inhibit neointimal hyperplasia through suppressing the NF- $\kappa \mathrm{B} / \mathrm{PCNA}$ signalling pathway.

\section{Discussion}

The first PTCA of a simple vascular lesion was performed in 1977 by Andreas Grüntzig (11). Since then, CAD treatment has come into a new world followed by the evolution of percutaneous interventions (2), but restenosis has been a tough problem. Recently, DES and DCB have been hot topics among researchers.

The pathogenesis of restenosis is multifactorial, involving such events as endothelial injury, inflammation, platelet activation, and hyperplasia of the intima, primarily due to vascular smooth muscle cell (VSMC) replication (12). DES or DCB delivers drugs into the vessel wall, where they would remain for a while to work. Due to the high cost of imported paclitaxel-coated balloons, we have been committed to finding another drug to help more patients.

TMP is a traditional Chinese medicine frequently used in clinical practice to assist treatment of circulatory diseases. It suppresses VSMC proliferation, and platelet aggregation, and protects endothelial cells. As previously reported (13), TMP might suppress the expression of c-fos and c-myc proliferation genes; thus, VSMCs of New-Zealand White rabbits in the G1 phase increased greatly, and those in $\mathrm{S}$ and G2-M phase decreased, which meant that proliferation of vascular smooth muscle was inhibited simultaneously with the action time. A study suggested that TMP inhibited Ang II induced proliferation and collagen secretion of VSMCs through down-regulating the Wnt signal pathway (14). In addition, some studies showed that TMP blocked the PKC and ERK1/2 pathway and suppressed AP-1 and PCNA expression (15). In addition, a study indicated that TMP inhibits HG-induced oxidative injury and VSMC proliferation by inhibiting ROS overproduction and p38MAPK/JNK/ERK phosphorylation (16). In our previous study, TES prevented neointimal hyperplasia and in-stent restenosis in a porcine model by reducing late lumen loss, DS\%, and NA, and increasing LA (9). Another study illustrated that TMP had anti-platelet effects dependent on time and dosage in rats. In addition, TMP might block calcium channels to loosen contractile Salganicoff human platelet strips (17). More studies found that TMP prevented platelet aggregation and thrombosis by inhibiting thrombin and ADP and protecting endothelia
$(18,19)$. Moreover, Li's study revealed that TMP could inhibit LPS-induced IL-8 production mediated by the NF$\kappa B-$ dependent pathway (20). It also could downregulate PDGF-BB-induced VSMC proliferation and migration partly, at least, through inhibiting the activation of the ERK and P38 MAPK signalling (21). Owing to these confirmed features and effects of TMP, we sprayed TMP onto balloons and delivered the drug into vessel lesions to evaluate its function in restenosis.

To imitate artery conditions as closely to those of humans as possible, we designed a local stenosis model of the rabbit iliac artery by injuring iliac artery endothelial cells with balloons. To improve the model, we chose balloons with diameters greater than normal rabbit target vessels by approximately $30-40 \%$, according to documented records and diameters of experimental rabbits' vessels, to ensure sufficient damage to endothelia and provided high-fat diets to simulate the internal condition of real patients. Then, we used models treated with plain balloons as controls to compare with TCBs. Four weeks after angioplasty, we found that stenosis was more severe in the PBA group than in the TCB group. On angiograms, the TCB group had larger MLD and relatively smaller DS\%; on histological sections, the TCB group had obviously larger LA and smaller AS\%.

This study had some limitations. First, the rabbit model was artificially developed with balloons, which cannot be equated to a human atherosclerotic artery and internal environment. Second, we chose uninjured iliac arteries in group B as reference arteries. Considering the response of the whole body, we cannot guarantee that the uninjured arteries were totally normal, which might cause underestimation of the percentage of luminal narrowing in restenotic lesions (4). Third, the TCBs were produced by us, so the quality of balloons had certain defects and need greater optimization. Fourth, we did not detect possible molecules, so we cannot confirm further mechanisms of TMP. Therefore, more experiments are required for in-depth study of the mechanisms of TCBs and to improve TCBs.

TCB efficiently mitigated restenosis in a rabbit model of iliac artery balloon injury. It might restrain intimal hyperplasia of vessels by inhibiting the activation of the $\mathrm{NF}-\kappa \mathrm{B}$ pathway to reduce the inflammatory response, and decreasing the rate of cell proliferation through suppressing PCNA expression. TCB can be a superior alternative to certain conditions in the treatment of CAD to effectively and economically prevent restenosis. 


\section{Acknowledgments}

Funding: The National Natural Science Foundation of China $(81270203,81770231)$, the science and technology development project of Nanjing (201303048), the Natural Science Foundation of Jiangsu (BK20161436), Jiangsu Provincial Key Medical Discipline (Laboratory ZDXKA2016023), and Jiangsu Provincial Key Research and Development Program (BE2016785). The funders had no role in the whole study design, data collection and analysis or preparation of the manuscript.

\section{Footnote}

Conflicts of Interest: The authors have no conflicts of interest to declare.

Ethical Statement: The authors are accountable for all aspects of the work in ensuring that questions related to the accuracy or integrity of any part of the work are appropriately investigated and resolved. Animal experimental ethical inspection form of Southeast University (No. 20160114003).

\section{References}

1. Bauters C, Isner JM. The biology of restenosis. Prog Cardiovasc Dis 1997;40:107-16.

2. Bhargava B, Karthikeyan G, Abizaid AS, et al. New approaches to preventing restenosis. BMJ 2003;327:274-9.

3. Weintraub WS. The pathophysiology and burden of restenosis. Am J Cardiol 2007;100:3K-9K.

4. Kakuta T, Usui M, Coats WD Jr, et al. Arterial remodeling at the reference site after angioplasty in the atherosclerotic rabbit model. Arterioscler Thromb Vasc Biol 1998;18:47-51.

5. Picard F, Doucet S, Asgar AW. Contemporary use of drugcoated balloons in coronary artery disease: Where are we now? Arch Cardiovasc Dis 2017;110:259-72.

6. Benezet J, Agarrado A, Gutierrez-Barrios A, et al. Comparison of two different drug-coated balloons for the treatment of in-stent restenosis: A long-term single-centre experience. Cardiovasc Revasc Med 2016;17:176-80.

7. Bondesson P, Lagerqvist B, James SK, et al. Comparison of two drug-eluting balloons: a report from the SCAAR registry. EuroIntervention 2012;8:444-9.

8. Nijhoff F, Stella PR, Troost MS, et al. Comparative assessment of the antirestenotic efficacy of two paclitaxel drug-eluting balloons with different coatings in the treatment of in-stent restenosis. Clin Res Cardiol 2016;105:401-11.

9. Ma G, Ding S, Feng Y, et al. Tetramethylpyrazine-eluting stents prevented in-stent restenosis in a porcine model. J Cardiovasc Pharmacol 2007;50:201-5.

10. Yamamoto E, Watanabe S, Bao B, et al. Preclinical evaluation of a paclitaxel-incorporated nanoparticle-coated balloon in rabbit and porcine models. Cardiovasc Revasc Med 2018;19:433-7.

11. Grüntzig AR, Senning A, Siegenthaler WE. Nonoperative dilatation of coronary-artery stenosis: percutaneous transluminal coronary angioplasty. $\mathrm{N}$ Engl J Med 1979;301:61-8.

12. Casscells W. Migration of smooth muscle and endothelial cells. Critical events in restenosis. Circulation 1992;86:723-9.

13. Li G, Zhang Y, Chen Q.Effect of Tetramethylpyrazine on vascular smooth muscle cell proliferation induced by thrombin. Chinese Heart Journal 2007;19:646-8.

14. Hua JY, He YZ, Jiang XH, et al. Effect of Tetramethylpyrazine on the Proliferation and Collagen Synthesis of Vascular Smooth Muscle Cells. Zhongguo Zhong Xi Yi Jie He Za Zhi 2013;33:1226-31.

15. Sun YP, Wang X, Zhang DW, et al. Effects of Tetramethylpyrazine on PKC -ERK1/2 Signal Pathway in Proliferation of Vascular Smooth Muscle Cells Induced by AngII. Lishizhen Medicine \& Materia Medica Research 2009;20:1654-5.

16. Liu Y, Li X, Jiang S, et al. Tetramethylpyrazine protects against high glucose-induced vascular smooth muscle cell injury through inhibiting the phosphorylation of JNK, p38MAPK, and ERK. J Int Med Res 2018;46:3318-26.

17. Zhou XB, Salganicoff L, Sevy R. The pharmacological effect of ligustrazine on human platelets. Acta Pharmaceutica Sinica 1985;20:334-9.

18. Li M, Zhao MQ, Kumar Durairajan SS, et al. Protective effect of tetramethylpyrazine and salvianolic acid B on apoptosis of rat cerebral microvascular endothelial cell under high shear stress. Clin Hemorheol Microcirc 2008;38:177-87.

19. Zhang F, Ni C, Kong D, et al. Ligustrazine attenuates oxidative stress-induced activation of hepatic stellate cells by interrupting platelet-derived growth factor- $\beta$ receptormediated ERK and p38 pathways. Toxicol Appl Pharmacol 2012;265:51-60.

20. Li XY, He JL, Liu HT, et al. Tetramethylpyrazine suppresses interleukin-8 expression in LPS-stimulated human umbilical vein endothelial cell by blocking ERK, 
p38 and nulear factor-kappaB signaling pathways. J

Ethnopharmacol 2009;125:83-9.

21. Yu L, Huang X, Huang K, et al. Ligustrazine attenuates the platelet-derived growth factor-BB-induced

Cite this article as: Chen L, Pang S, Hao C, Xie A, Zhu K, He Y, Zhang X, Lu W, Ma G, Chen Z. Prevention of neointimal formation after angioplasty using tetramethylpyrazine-coated balloon catheters in a rabbit iliac artery model. Cardiovasc Diagn Ther 2019;9(5):472-480. doi: 10.21037/cdt.2019.09.04 proliferation and migration of vascular smooth muscle cells by interrupting extracellular signal-regulated kinase and P38 mitogen-activated protein kinase pathways. Mol Med Rep 2015;12:705-11. 